\title{
A case report of advanced thymoma re-treated with PD-1 inhibitor after initial immune-related pneumonitis
}

\author{
Yao Zhang ${ }^{1,2 \#} \wedge$, Wenhua $\mathrm{Li}^{1,2 \#}$, Xichun $\mathrm{Hu}^{1}$, Jian Zhang ${ }^{1}$ \\ ${ }^{1}$ Department of Medical Oncology, Fudan University Shanghai Cancer Center, Shanghai, China; ${ }^{2}$ Department of Oncology, Shanghai Medical \\ College, Fudan University, Shanghai, China \\ \#These authors contributed equally to this work. \\ Correspondence to: Jian Zhang, MD, PhD. Department of Medical Oncology, Fudan University Shanghai Cancer Center, 270 Dong'an Road, Shanghai, \\ China; Department of Oncology, Shanghai Medical College, Fudan University, 138 Yixueyuan Road, Shanghai, China. Email: syner2000@163.com.
}

\begin{abstract}
With the growing use of immune checkpoint inhibitors (ICIs), recurrent immune-related adverse events (irAEs) have become more common after recovery from initial immune toxicity. Here we report one case of anti-PD-1-related pneumonitis in a patient with advanced thymoma, who experienced two episodes of pneumonitis during 10 months of treatment with a PD-1 inhibitor. By reviewing recent advances in ICIrelated adverse events, we summarize the clinical characteristics of recurrent immune-related pneumonitis, illustrate potential predictive biomarkers for irAEs, and evaluate the value of resuming anti-PD-1 treatment in this patient. To date, evidence regarding retreatment following improvement from an irAE is scarce, and further clinical trials are needed to address this scenario.
\end{abstract}

Keywords: Immune-related adverse event (irAE); recurrent pneumonitis; anti-PD-1; case report

Submitted Apr 18, 2021. Accepted for publication Aug 18, 2021.

doi: 10.21037/apm-21-943

View this article at: https://dx.doi.org/10.21037/apm-21-943

\section{Introduction}

To date, the FDA has approved six immune checkpoint inhibitors (ICIs) targeting the PD-1/PD-L1 pathway for treating advanced tumors. Compared with chemotherapy, PD(L)-1 inhibitors have a relatively modest toxicity profile. However, the toxic effects of monoclonal antibodies against immune checkpoint receptors may affect any organ, including the lungs, colon, thyroid, skin, pituitary, heart, and nervous system $(1,2)$. Pneumonitis is the leading cause of anti-PD(L)1-related death and discontinuation of ICIs $(3,4)$. Most immune-related adverse events (irAEs) are manageable with corticosteroids or other immune modulators. According to the ESMO guideline, a checkpoint inhibitor can be reintroduced after the daily dose of steroids is less than or equal to $10 \mathrm{mg}$ of oral prednisone per day for grades 1-2 (5). Recurrent or new immune-related irAEs have been documented in several studies (4,6-9). Naidoo et al. reported that the incidence of recurrent pneumonitis was up to $25 \%$ among patients who were rechallenged with ICIs (10). Herein, we present one case of recurrent immune-related pneumonitis to highlight the clinical characteristics of recurrent irAEs and answer several frequently asked questions about recognizing and managing this condition.

We present the following article following the CARE reporting checklist (available at https://dx.doi.org/10.21037/ apm-21-943).

All procedures performed in studies involving human participants were in accord with the ethical standards of the institutional and/or national research committee(s) and with the Helsinki Declaration (as revised in 2013). Written informed consent was obtained from the patient for

$\wedge$ ORCID: 0000-0003-1174-7049. 

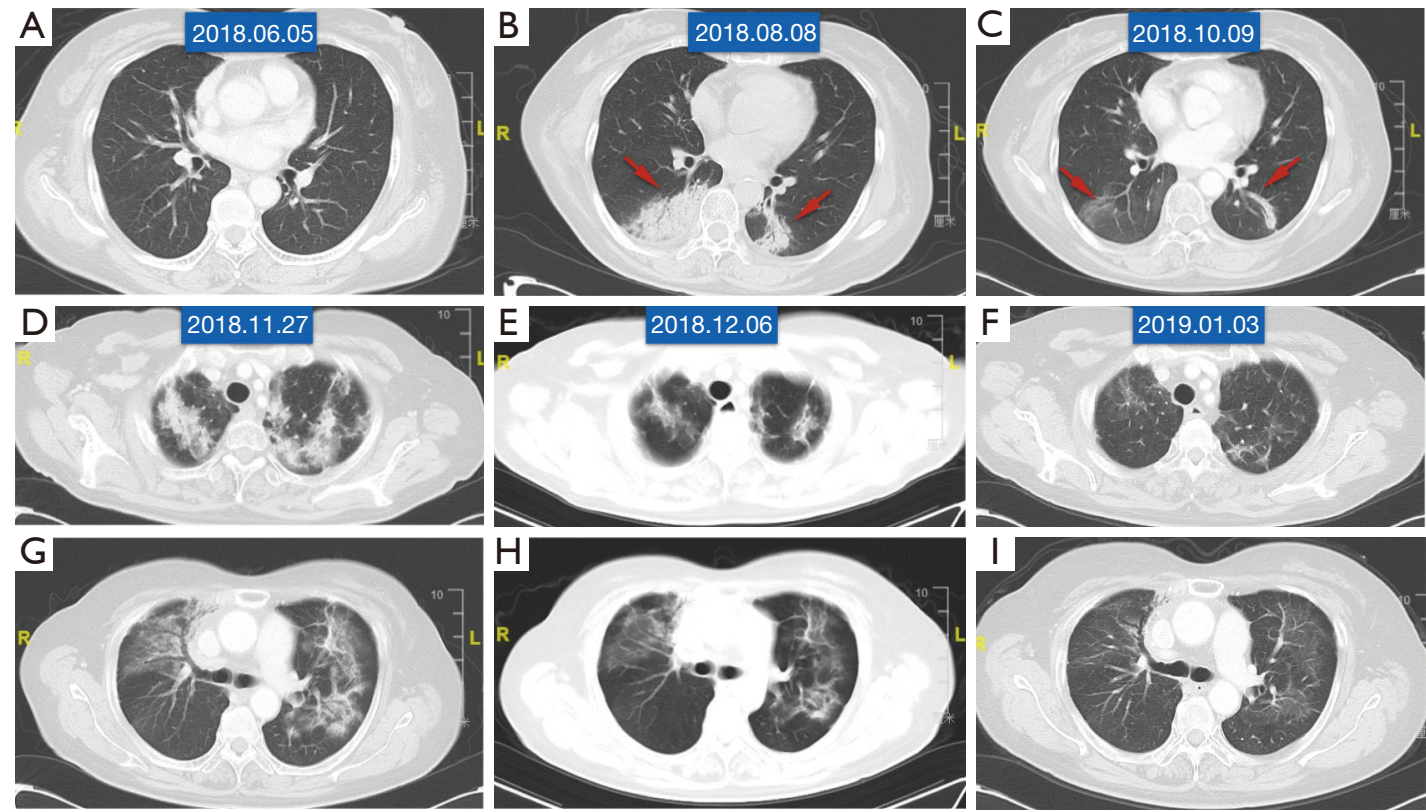

Figure 1 Chest CT scans demonstrate the clinical course of the two episodes of immune-related pneumonitis in this case example. (A) Chest CT before initiation of PD-1 inhibitor treatment. (B) Image of pneumonitis before prednisone treatment shows consolidation lesions involving the bilateral lower lobes with air bronchograms (red arrow). (C) Image captured after 6 weeks of prednisone treatment reveals resolution of opacities. (D,G) Chest CT following onset of the second pneumonitis shows GGO-like lesions involving multiple bilateral lobes with air bronchograms. (E,H) Images after 1 week of prednisone resumption demonstrate improvements in the second pneumonitis. (F,I) CT scans show the second pneumonitis has resolved with prednisone. CT, computed tomography; GGO, ground-glass opacity.

publication of this case report and accompanying images. A copy of the written consent is available for review by the editorial office of this journal.

\section{Case vignette}

This case was a 62 -year-old woman diagnosed with resected thymoma, metastatic to the lungs, subpleural, and mediastinum. She did not smoke or drink alcohol. There was no surgical history, no other prior diseases, and no family history of malignant diseases. She underwent adjuvant concomitant radiochemotherapy-60GY/30FX combined with four cycles of cisplatin and etoposide. The PE regimen was ceased for 5 months due to severe leukocytopenia (grade 3). She was then treated with two cycles of a TC regimen (paclitaxel + carboplatin). However, the patient's disease progressed despite these therapies. Treatment was initiated with a PD-1 inhibitor $200 \mathrm{mg}$ every 3 weeks. After five administrations, the patient reported malaise, cough, and dyspnea. Upon physical examination, she had an oxygen saturation of $98 \%$ in an open environment; the remainder of the physical examination was normal. Her serum C-reactive protein (CRP) level was $6.6 \mathrm{mg} / \mathrm{L}$ (normal range, $0-5 \mathrm{mg} / \mathrm{L}$ ). The blood count showed a normal white blood cell (WBC) count. The serum procalcitonin (PCT) level was normal. A chest computed tomography (CT) evidenced patchy bilateral consolidation involving the lower lobes without signs of pulmonary thromboembolism (Figure $1 A, 1 B)$. next-generation sequencing (NGS)based detection (11) ruled out causative pathogens. She was diagnosed with grade 2 ICI-related pneumonitis. Corticosteroid therapy was introduced with prednisone $1 \mathrm{mg} / \mathrm{kg}$ per day, tapering to $5-10 \mathrm{mg} /$ week for 5 weeks. The controlled CT scan after corticosteroid therapy showed almost full resolution of pulmonary opacities (Figure 1C). The anti-PD-1 inhibitor was then resumed in this patient. After three doses of PD-1 inhibitor retreatment, the patient reported a nonproductive cough and shortness of breath. On physical examination, her blood pressure was 123/81 $\mathrm{mmHg}$, her pulse was 117 beats per minute, her temperature was $37.3^{\circ} \mathrm{C}$, and her pulse oximetry was $94 \%$. The blood count result was normal. The serum CRP 
Table 1 Clinical tests were performed to rule out etiologies other than immune-related pneumonitis

\begin{tabular}{lll}
\hline Test & Results- ${ }^{\text {st }}$ episode & Results- $2^{\text {nd }}$ episode \\
\hline NGS-based pathogen detection & No causative organism detected & N/A \\
WBC counts & Normal & Normal \\
CRP & $6.6 \mathrm{mg} / \mathrm{L}(0-5 \mathrm{mg} / \mathrm{L})$ & $34.8 \mathrm{mg} / \mathrm{L}(0-5 \mathrm{mg} / \mathrm{L})$ \\
PCT & Normal & Normal \\
Chest CT & Bilateral patchy consolidation involving lower lobes & Bilateral GGO with air bronchograms \\
& without signs of pulmonary thromboembolism & involving multiple lobes \\
\hline
\end{tabular}

NGS, next-generation sequencing; N/A, not available; WBC, white blood cell; CRP, C-reactive protein; PCT, procalcitonin; CT, computed tomography; GGO, ground-glass opacities.

level was $34.8 \mathrm{mg} / \mathrm{L}$ (Table 1). A CT scan showed bilateral ground-glass opacities with air bronchograms involving multiple lobes (Figure 1D,1G). She was re-treated with prednisone at a dose of $1 \mathrm{mg} / \mathrm{kg}$ per day. The controlled CT performed 5 weeks after prednisone treatment showed significant improvement (Figure 1F,1I). However, according to the latest response evaluation, her thymoma continued to progress.

\section{Discussion}

The incidence of recurrent irAEs is rare. In a recent retrospective analysis conducted by Simonaggio et al. (4), 13 patients experienced initial immune-related pneumonitis, and five patients were rechallenged with the same $\mathrm{PD}(\mathrm{L})-1$ inhibitors. Of the rechallenged group, one patient (20\%) experienced a second pneumonitis. Pollack et al. (8) reported that $33 \%$ of patients ( 1 in 3 ) with pneumonitis experienced recurrence. Naidoo et al. (10) identified 12 patients rechallenged with immunotherapy after complete clinical resolution of initial pneumonitis, three $(25 \%)$ of whom experienced recurrent pneumonitis. In another retrospective study (7), 482 patients with lung cancer were treated with anti-PD(L)-1 ( \pm CTLA-4). Of the patients who developed irAEs, 26\% (10/39) experienced recurrent irAEs but improved after retreatment with anti-PD(L)1. Two out of six patients $(33 \%)$ with pneumonitis had recurrent or new irAEs.

In our patient, we wondered if any predisposing factors caused the recurrence of the immune-related pneumonitis. Pollack et al. (8) assessed whether the length and type of immunosuppressive treatment, the severity of initial toxicity, presence of ongoing steroids, symptoms at the anti-PD-1 resumption, or delay in resuming anti-PD-1 influenced the recurrence rate of irAEs in patients with advanced melanoma treated with anti-PD-1. They found that the duration between the final dose of CTLA-4 and PD-1 blockade to the resumption of anti-PD-1 was a little longer in patients without toxicities than those who experienced toxicities (median 62 days versus 56 days, $\mathrm{P}=0.03$ ). In our patient, the gap between the final dose of anti-PD-1 and the resumption of anti-PD-1 was 61 days. Delaying the PD-1 inhibitor retreatment after recovery from the first episode of pneumonitis may have reduced her risk of pneumonitis-flare. Nevertheless, the optimal time frame for the resumption of treatment should be confirmed by further studies. Other evidence revealed that anti-PD-1-related pneumonitis occurred more frequently in patients with a history of thoracic radiation than those without $(6.0 \%$ versus $2.6 \%)$, consistent with our case.

In Pollack's study (8), the same irAE that caused the initial ICI treatment discontinuation reoccurred in 14 (18\%) patients at a median of 14 days following ICI resumption (range, 7-167 days). The second episode of pneumonitis in our case occurred 49 days after ICI resumption. Symptoms of pneumonitis include nonproductive cough and dyspnea (10). Fever, chest pain, and hypoxia are less common, while onethird of patients are reportedly asymptomatic (12).

Although cases with recurrent irAEs are uncommon, the absolute number of patients experiencing irAEs is likely to increase as the use of anti-PD(L)-1 becomes more widespread. Therefore, biomarkers to predict which patients will experience immune-related toxicities are needed. Here we summarize some of the candidate markers from current evidence (Table 2).

\section{Radiomics}

Radiomics is a new field that provides comprehensive visualization and characterization of the tissue of interest 
Table 2 Potential biomarkers predicting anti-PD-1 toxicity

\begin{tabular}{|c|c|c|}
\hline Type & Biomarkers & Association \\
\hline \multirow[t]{4}{*}{ Blood } & $\begin{array}{l}\text { Serum antibody } \\
\text { profiles }\end{array}$ & $\begin{array}{l}\text { A subset of toxicity-associated antibodies could identify patients at risk of irAEs from } \\
\text { immunotherapy }\end{array}$ \\
\hline & Circulating B cells & $\begin{array}{l}\text { Early changes in the frequency of circulating B cells in patients treated with anti-CTLA-4 and } \\
\text { anti-PD-1 could be associated with an increased risk of irAEs }\end{array}$ \\
\hline & WBC and RLC & $\begin{array}{l}\text { An increased WBC count and a decreased RLC count have been associated with nivolumab-related } \\
\text { G3/4 and lung/GI irAEs }\end{array}$ \\
\hline & CXCL2 & $\begin{array}{l}\text { Changes in the plasma CXCL2 level have been significantly associated with nivolumab-related irAEs } \\
\text { in NSCLC patients }\end{array}$ \\
\hline Tissue & T cell repertoire & A significant overlay of T cell clones infiltrating pneumonitis lesions and tumors has been identified \\
\hline
\end{tabular}

irAEs, immune-related adverse events; WBC, white blood cell; RLC, relative lymphocyte cell; CXCL2, chemokine ligand 2; NSCLC, nonsmall cell lung cancer; $\mathrm{ICI}$, immune checkpoint inhibitor.

and the related microenvironment by automated extraction of high fidelity, high-dimensional medical imaging features from standard images. According to Sun et al. (13), radiomics could be useful in predicting clinical outcomes of patients treated with immunotherapy. Furthermore, Colen et al. (14) performed a radiomic analysis of baseline chest computed tomography (CT) images of patients who did. It did not develop ICI-induced pneumonitis and constructed a one-way algorithm to predict which patients were likely to experience ICI-induced pneumonitis successfully. Although the study was retrospective, included only two cases and 30 controls, and lacked external validation, it demonstrated a possible, non-invasive way to predict irAEs, especially pneumonitis. The results of their prospective studies are expected.

\section{Baseline serum antibody profile}

Gowen et al. (15) identified a panel of specific antibodies differentially expressed in patients with severe anti-PD-1related toxicity. Table 3 summarizes the top 10 differentially expressed antibodies, four of which are highly expressed in brain tissues and two of which are generally expressed in most tissues. However, this study did not specifically address pneumonitis-related antibodies or other specific irAErelated antibodies. A more detailed analysis of pneumonitis and further independent validation are needed in a clinical trial setting.

\section{Circulating B cells}

Liudahl et al. (16) found that patients with a $30 \%$ or more decrease in baseline levels of total circulating B cells and a twofold or greater elevation in CD2 $1^{\text {lo }} \mathrm{B}$ cells or plasmablasts were more susceptible to high-grade irAEs than patients without $\mathrm{B}$ cell alterations.

\section{Routine blood counts}

A retrospective study showed that an increased WBC count and a decreased relative lymphocyte cell (RLC) count were associated with nivolumab-related G3/4 and lung/GI irAEs (17). In our patient, a decrease in the RLC count was noticed at the time of the second episode of pneumonitis, without an abnormal WBC count (Figure $2 A, 2 B$ ). In our view, the routine peripheral blood count is affected by many factors, such as the use of glucocorticoids, which lowers its reliability as a potential marker.

\section{Chemokine ligand 2 (CXCL2)}

The plasma level of CXCL2 was found to be related to nivolumab-associated irAEs in non-small cell lung cancer (NSCLC) patients (18). In a cohort study, Matsuo et al. found that an elevation of CXCL2 above the baseline was significantly correlated with disease progression. 
Table 3 Top 10 differentially expressed antibodies associated with anti-PD-1-related toxicity

\begin{tabular}{ll}
\hline Name & Protein expression \\
\hline NECAP1 & Highly expressed in the brain \\
NRCAM & Highly expressed in cerebrospinal fluid and the brain \\
PEX19 & Highly expressed in the pancreas and bones \\
PSMD4 & Expressed in most tissues \\
C6orf106 & General cytoplasmic and membranous expression \\
SLC2A11 & Highly expressed in the retina \\
ZBTB21 & Highly expressed in the brain and adrenal \\
RCAN3 & Highly expressed in CD4 T cells, lymph nodes, peripheral blood mononuclear cells, and CD8 T cells \\
AP2M1 & Highly expressed in the brain \\
ING3 & Highly expressed in CD8 T cells and peripheral blood mononuclear cells \\
\hline
\end{tabular}
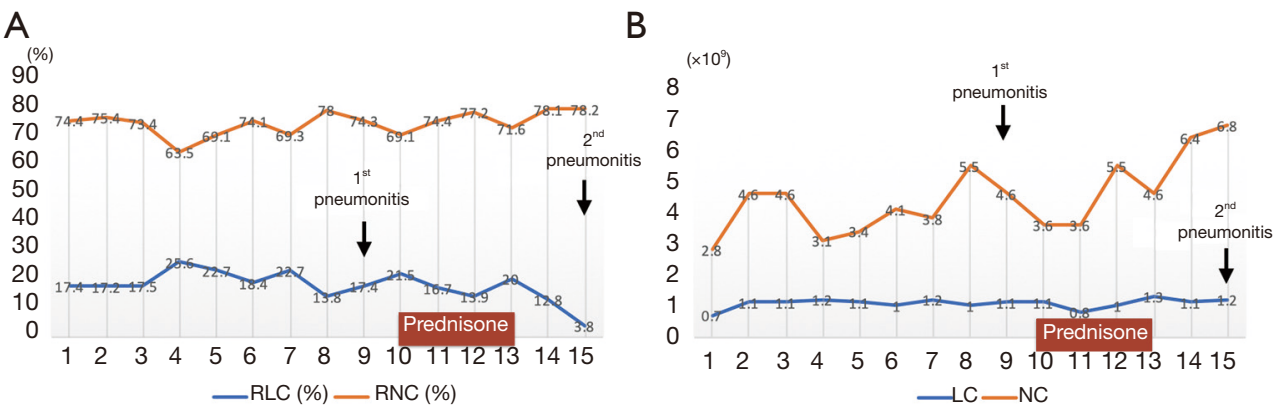

Figure 2 Routine blood tests in this case example. (A) Relative lymphocyte count (blue line) and relative neutrophil count (yellow line) of this patient: a slight decrease of RLC was noticed at the time of the second pneumonitis. (B) Absolute lymphocyte counts (blue line) and absolute neutrophil counts (yellow line) of this patient. RLC, relative lymphocyte cell.

\section{T cell repertoire}

By sequencing the $T$ cell receptor, Läubli et al. (19) found that irAEs lesions in patients under PD-1 blockade were infiltrated by $\mathrm{T}$ cells with a similar specificity as tumorinfiltrating $\mathrm{T}$ cells.

\section{MicroRNA-146a}

Reduced microRNA-146a was found to be associated with an increased risk of developing irAEs (20).

Since no prospective trials have defined the best treatment approaches for ICI-related pneumonitis recurrence, patients are generally treated as per the initial pneumonitis following the guidelines published by American Society of Clinical Oncology (ASCO), European Society for Medical Oncology (ESMO), or Society for Immunotherapy of
Cancer (SITC). This case report presents the clinical course, diagnostic evaluation, management, and outcome of antiPD-1-related recurrent pneumonitis. This patient was diagnosed with thymoma and was enrolled in a clinical trial of a PD-1 inhibitor. Though no $\mathrm{PD}(\mathrm{L})-1$ inhibitors have been recommended by National Comprehensive Cancer Network (NCCN) for thymoma (21), evidence showed that positive PD-L1 expressions were detected in thymomas (22), which implied that anti-PD-1/PD-L1 drugs could be of potential use in unresectable or relapsed thymomas. Indeed, a partial response was observed in this patient after four doses of PD-1 inhibitor (Figure 3A,3B), although followed by pneumonitis. She was sensitive to prednisone treatment. However, retreatment with the PD-1 inhibitor led to recurrent pneumonitis, even more severe on the CT scan than the initial pneumonitis.

Although some experts do not recommend retreatment 

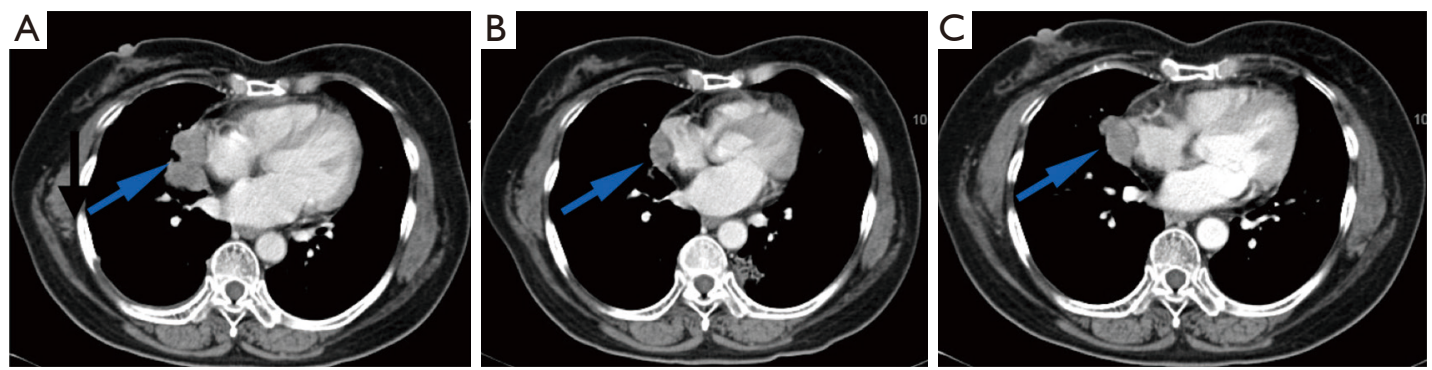

Figure 3 Contrast-enhanced CT scans demonstrate a partial response in this case example. (A) Chest CT prior to the treatment with the PD-1 inhibitor shows a right upper lobe mass (blue arrow). (B) Chest CT after four doses of the PD-1 inhibitor shows a $30 \%$ decrease in the diameter of the target lesion. (C) After discontinuing the PD-1 inhibitor for 2 months, chest CT shows the size of the target lesion has increased by at least $20 \%$. CT, computed tomography.

with ICIs, no current evidence supports this opinion. Several studies have found that most recurrent toxicities due to immunosuppressant drugs are remediable. We believe that retreatment with PD-1 inhibitors can be carefully considered in some patients with irAEs.

Santini et al. (7) found that the efficacy analysis of patients who had an objective response before the onset of an irAE was similar in the retreatment and discontinuation cohorts. Our patient had a PR before the first episode of pneumonitis and resumed the PD-1 inhibitor for three doses after recovering from the irAE. Her disease progressed following the second pneumonitis, with the size of the target lesion increasing over 20\% (Figure 3C). The patient would not have benefited from further anti-PD-1 inhibitor retreatment based on recent evidence and expert consensus. Thus, anti-PD-1 treatment was permanently discontinued in this patient.

Several studies have reported a positive association between the development of irAEs and clinical outcomes in patients treated with PD- 1 inhibitors or CTLA-4 inhibitors $(23,24)$. Das et al. (25) summarized several clinical studies showing the outcomes of patients with and without irAEs in each study. Interestingly, most of the studies reported a better objective response rate (ORR) in patients with irAEs. However, the underlying molecular mechanisms between irAEs, or some specific irAEs, and clinical efficacy have not been fully uncovered.

\section{Conclusions}

This case report provides a detailed clinical course of recurrent ICI-related pneumonitis. By reviewing the "state of the art" literature, we summarize the clinical characteristics of this rare but fatal irAE, list the potential biomarkers and management guidelines for ICI-related pneumonitis, and discuss the value of ICI resumption for patients with irAEs. The biomarkers above may predict anti-PD-1-related toxicity in patients. However, there is a long way to go before the routine application of ICI retreatment in clinical practice. First, the efficacy of retreatment should be verified in larger, prospective studies. Since the mechanisms of irAEs are poorly understood, further studies will help us find more biomarkers to define patients who will benefit most from these effective but potentially toxic drugs. Furthermore, more clinical data will enable treating clinicians to select appropriate patients who would benefit from ICI resumption after initial toxicities.

\section{Acknowledgments}

Funding: None.

\section{Footnote}

Reporting Checklist: The authors have completed the CARE reporting checklist. Available at https://dx.doi. org/10.21037/apm-21-943

Conflicts of Interest: All authors have completed the ICMJE uniform disclosure form (available at https://dx.doi. org/10.21037/apm-21-943). The authors have no conflicts of interest to declare.

Ethical Statement: The authors are accountable for all aspects of the work in ensuring that questions related to the accuracy or integrity of any part of the work are 
appropriately investigated and resolved. All procedures performed in studies involving human participants were in accordance with the ethical standards of the institutional and/or national research committee(s) and with the Helsinki Declaration (as revised in 2013). Written informed consent was obtained from the patient for publication of this case report and accompanying images. A copy of the written consent is available for review by the editorial office of this journal.

Open Access Statement: This is an Open Access article distributed in accordance with the Creative Commons Attribution-NonCommercial-NoDerivs 4.0 International License (CC BY-NC-ND 4.0), which permits the noncommercial replication and distribution of the article with the strict proviso that no changes or edits are made and the original work is properly cited (including links to both the formal publication through the relevant DOI and the license). See: https://creativecommons.org/licenses/by-nc-nd/4.0/.

\section{References}

1. Postow MA, Sidlow R, Hellmann MD. Immune-Related Adverse Events Associated with Immune Checkpoint Blockade. N Engl J Med 2018;378:158-68.

2. Abu-Sbeih H, Ali FS, Naqash AR, et al. Resumption of Immune Checkpoint Inhibitor Therapy After ImmuneMediated Colitis. J Clin Oncol 2019;37:2738-45.

3. Wang DY, Salem JE, Cohen JV, et al. Fatal Toxic Effects Associated With Immune Checkpoint Inhibitors: A Systematic Review and Meta-analysis. JAMA Oncol 2018;4:1721-8.

4. Simonaggio A, Michot JM, Voisin AL, et al. Evaluation of Readministration of Immune Checkpoint Inhibitors After Immune-Related Adverse Events in Patients With Cancer. JAMA Oncol 2019;5:1310-7.

5. Haanen JBAG, Carbonnel F, Robert C, et al. Management of toxicities from immunotherapy: ESMO Clinical Practice Guidelines for diagnosis, treatment and followup. Ann Oncol 2017;28:iv119-42.

6. Nishino M, Chambers ES, Chong CR, et al. Anti-PD-1 Inhibitor-Related Pneumonitis in Non-Small Cell Lung Cancer. Cancer Immunol Res 2016;4:289-93.

7. Santini FC, Rizvi H, Plodkowski AJ, et al. Safety and Efficacy of Re-treating with Immunotherapy after Immune-Related Adverse Events in Patients with NSCLC. Cancer Immunol Res 2018;6:1093-9.

8. Pollack MH, Betof A, Dearden H, et al. Safety of resuming
anti-PD-1 in patients with immune-related adverse events (irAEs) during combined anti-CTLA-4 and anti-PD1 in metastatic melanoma. Ann Oncol 2018;29:250-5.

9. Abou Alaiwi S, Xie W, Nassar AH, et al. Safety and efficacy of restarting immune checkpoint inhibitors after clinically significant immune-related adverse events in metastatic renal cell carcinoma. J Immunother Cancer 2020;8:e000144.

10. Naidoo J, Wang X, Woo KM, et al. Pneumonitis in Patients Treated With Anti-Programmed Death-1/ Programmed Death Ligand 1 Therapy. J Clin Oncol 2017;35:709-17.

11. Ye $M$, Wei $W$, Yang $Z$, et al. Rapid diagnosis of Propionibacterium acnes infection in patient with hyperpyrexia after hematopoietic stem cell transplantation by next-generation sequencing: a case report. BMC Infect Dis 2016;16:5.

12. Chuzi S, Tavora F, Cruz M, et al. Clinical features, diagnostic challenges, and management strategies in checkpoint inhibitor-related pneumonitis. Cancer Manag Res 2017;9:207-13.

13. Sun R, Limkin EJ, Vakalopoulou M, et al. A radiomics approach to assess tumour-infiltrating CD8 cells and response to anti-PD-1 or anti-PD-L1 immunotherapy: an imaging biomarker, retrospective multicohort study. Lancet Oncol 2018;19:1180-91.

14. Colen RR, Fujii T, Bilen MA, et al. Radiomics to predict immunotherapy-induced pneumonitis: proof of concept. Invest New Drugs 2018;36:601-7.

15. Gowen MF, Giles KM, Simpson D, et al. Baseline antibody profiles predict toxicity in melanoma patients treated with immune checkpoint inhibitors. J Transl Med 2018;16:82.

16. Liudahl SM, Coussens LM. B cells as biomarkers: predicting immune checkpoint therapy adverse events. J Clin Invest 2018;128:577-9.

17. Fujisawa Y, Yoshino K, Otsuka A, et al. Fluctuations in routine blood count might signal severe immunerelated adverse events in melanoma patients treated with nivolumab. J Dermatol Sci 2017;88:225-31.

18. Matsuo N, Azuma K, Hattori S, et al. Inflammatory biomarkers for prediction of tumor responses and adverse events in non-small cell lung cancer patients treated with anti-PD-1 inhibitor. J Clin Oncol 2018;36:e21050.

19. Läubli H, Koelzer VH, Matter MS, et al. The T cell repertoire in tumors overlaps with pulmonary inflammatory lesions in patients treated with checkpoint inhibitors. Oncoimmunology 2018;7:e1386362.

20. Marschner D, Falk M, Javorniczky NR, et al. MicroRNA- 
146a regulates immune-related adverse events caused by immune checkpoint inhibitors. JCI Insight 2020;5:132334.

21. Silva JL, De Moura Gallo CV, Costa DC, et al. Prion-like aggregation of mutant p53 in cancer. Trends Biochem Sci 2014;39:260-7.

22. Katsuya Y, Fujita Y, Horinouchi H, et al. Immunohistochemical status of PD-L1 in thymoma and thymic carcinoma. Lung Cancer 2015;88:154-9.

23. Judd J, Zibelman M, Handorf E, et al. Immune-Related Adverse Events as a Biomarker in Non-Melanoma Patients

Cite this article as: Zhang $\mathrm{Y}, \mathrm{Li}$ W, Hu X, Zhang J. A case report of advanced thymoma re-treated with PD-1 inhibitor after initial immune-related pneumonitis. Ann Palliat Med 2021;10(9):10083-10090. doi: 10.21037/apm-21-943
Treated with Programmed Cell Death 1 Inhibitors. Oncologist 2017;22:1232-7.

24. Rogado J, Sánchez-Torres JM, Romero-Laorden N, et al. Immune-related adverse events predict the therapeutic efficacy of anti-PD-1 antibodies in cancer patients. Eur J Cancer 2019;109:21-7.

25. Das S, Johnson DB. Immune-related adverse events and anti-tumor efficacy of immune checkpoint inhibitors. J Immunother Cancer 2019;7:306. 